\title{
Einführung zum Thema
}

Gynäkologe 2020 · 53:644

https://doi.org/10.1007/s00129-020-04681-5

(c) Springer Medizin Verlag $\mathrm{GmbH}$, ein Teil von Springer Nature 2020

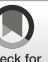

Check for
updates

Liebe Kolleginnen und Kollegen,

Sie halten das Leitthemenheft „Endometriose" in der Hand und sind vielleicht mit dem Leitthema etwas unglücklich? Dann sind Sie hier genau richtig!

Wenn Ihnen die Endometriose ein wenig ungeheuer ist und als "ChamäleonEntität" unseres Fachs nur schwer zu fassen scheint, wollen wir Ihnen mit diesem Heft einen neuen, hoffentlich möglichst praxisnahen Zugang zu dieser Erkrankung verschaffen.

Vielleicht werden wir nie genau verstehen, wie die Endometriose entsteht. Frau Prof. Mechsner et al. möchte Ihnen dennoch einen Überblick über die Bedeutung der Pathogenese für die im Alltag wichtigsten Probleme in Zusammenhang mit der Endometriose geben: die Schmerzentstehung und die Einschränkung der Fertilität. Im Beitrag von Prof. Meinhold-Heerlein et al. erfahren wir den aktuellen Stand zu den konservativen Behandlungsmöglichkeiten der Endometriose, die für die meisten von uns im praktischen Alltag das am meisten relevante Thema sein dürfte.

\section{》) Eine chirurgische}

Therapie sollte - ähnlich

wie in der Onkologie - in

Endometriosezentren erfolgen

Die Kollegen Schüring et al. widmen sich fokussiert noch einmal allen Aspekten der Subfertilität in Zusammenhang mit der Endometriose und bieten damit auch den perfekten Übergang zum Beitrag der Kollegen Schäfer et al. über die operative Therapie. Auch wenn die chirurgische Behandlung der Endometriose - ähnlich wie in der Onkologie - am besten in En-

Ludwig Kiesel ${ }^{1}$. Wolfgang Janni ${ }^{2}$

'Klinik für Frauenheilkunde und Geburtshilfe, Universitätsklinikum Münster, Münster, Deutschland

${ }^{2}$ Frauenklinik, Universitätsklinikum Ulm, Ulm, Deutschland

\section{Endometriose}

dometriosezentren durchgeführt werden sollte, um alle Therapiemöglichkeiten anbieten zu können, ist das Wissen um die Möglichkeiten für uns alle bedeutsam.

Der abschließende Beitrag von Krentel et al. ermöglicht es, unsere Einsichten zu der besonders herausfordernden Manifestation der Endometriose - in Form der Adenomyosis uteri - zu vertiefen.

Wir hoffen sehr, dass Sie nach Lektüre dieses Heftes neben der Herausforderung dieser Erkrankung auch die Faszination dieses besonderen Krankheitsbildes spüren können. Spiegelt die große Bandbreite der Aspekte der Endometriose schließlich nicht recht gut die Diversität unseres gesamten Fachgebietes wider? Die Frauenheilkunde ist immerhin eines der am breitesten gefächerten Gebiete in der der Medizin. Und genau das macht für viele von uns die Faszination unseres Faches aus - wie auch die bei der Behandlung der Endometriose.

Wir wünschen Ihnen eine erkenntnisreiche Lektüre,

mit herzlichen Grüßen aus Münster und Ulm

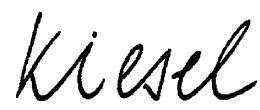

Ludwig Kiesel

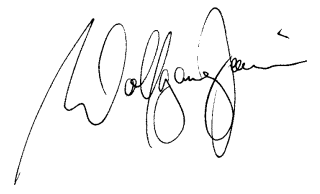

Wolfgang Janni

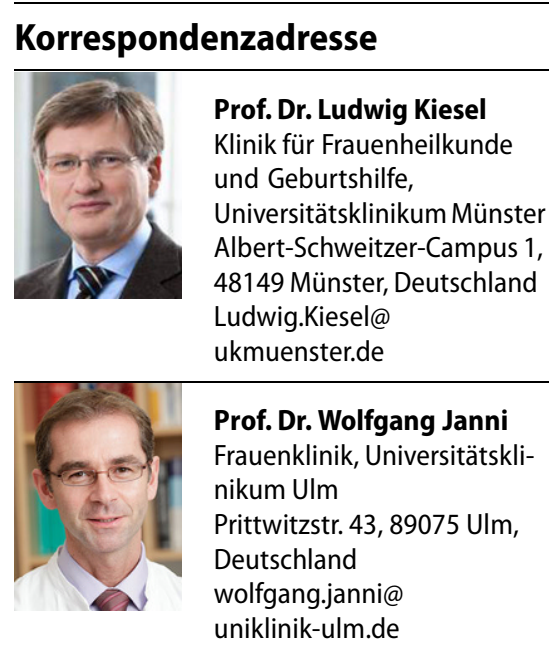

Interessenkonflikt. L. Kiesel und W. Janni geben an, dass kein Interessenkonflikt besteht. 